\title{
Intrinsic defects and the D1 to D4 optical bands detected in plastically deformed Si
}

\author{
R. Jones, B. J. Coomer, J. P. Goss \\ Department of Physics, University of Exeter, Exeter, EX4 4QL, United Kingdom \\ S. Öberg \\ Department of Mathematics, University of Luleå, Luleå S-97187, Sweden
}

\begin{abstract}
P. R. Briddon
Department of Physics, The University of Newcastle upon Tyne, Newcastle upon Tyne NE1 7RU, United Kingdom

(April 3, 2000)
\end{abstract}

\begin{abstract}
The properties of multi-vacancy and multi-interstitial defects that possess luminescent bands around $1 \mathrm{eV}$ are reviewed. Prominent among these are the hexavacancy and tri- and tetra- self-interstitial defects. It is suggested that the formation of these defects on dislocation cores could lead to the D1 to D4 photoluminescent bands linked to dislocations in Si and SiGe.
\end{abstract}




\section{INTRODUCTION}

The origin of the photoluminescent bands D1 to D4 at $0.807,0.870,0.935$ and $1.0 \mathrm{eV}$ connected with dislocations in silicon has been a major problem even after almost a quarter of century of investigation ${ }^{1}$. The recent observations ${ }^{2}$ of room temperature electroluminescence from D1 and D2 has spurred further activity. Many experiments have been carried out, and models advanced, but none has been universally accepted due in no small way to the difficulty of identifying point defects clustered at the dislocation core. It does seem likely that the reconstructed cores favoured by theory ${ }^{3}$ cannot lead to optical transitions as low as around $0.8 \mathrm{eV}$. Thus models based on gettered impurities like transition metals $^{4}$, oxygen ${ }^{5}$ or intrinsic defects like kinks and jogs ${ }^{6}$ have been proposed. The prevalence of the bands - seen in FZ and $\mathrm{Cz}$ n-Si and p-Si as well as SiGe - gives doubt that impurities are primarily responsible while kink and jog densities are sensitive to annealing conditions. Instead we propose here, that multivacancy and/or self-interstitial clusters trapped at the core may be responsible. Of course, such defects may arise from oxygen precipitation or from the growth of transition metal colonies and the origin of the luminescence misdiagnosed.

This hypothesis has grown out of recent investigations into optically active multiinterstitial and multi-vacancy point defects in Si. Our conclusion is that there are small aggregates, stable to $500^{\circ} \mathrm{C}$, which are optically active giving luminescent and absorption bands around $1 \mathrm{eV}$, and these results advance naturally the hypothesis that the $\mathrm{D}$ bands are due to similar vacancy and interstitial clusters trapped at the dislocation core.

\section{THE HEXAVACANCY}

Our investigations began by analysing the electrical properties of the hexavacancy in $\mathrm{Si}$. The ideal form of this defect contains, like other vacancy centres, dangling bonds which have a tendency to reconstruct in pairs. The best known example is the divacancy where four of the dangling bonds are paired yielding a defect with $C_{2 h}$ symmetry and whose electrical activity largely emanates from the two remaining Si dangling bonds lying in the mirror plane ${ }^{7}$. These dangling bonds give acceptor levels $(-/ 0)$ and $(=/-)$ at $E_{c}-0.43$ and $E_{c}-0.23 \mathrm{eV}$ respectively. There is also a donor level at $E_{v}+0.19 \mathrm{eV}$. The $\mathrm{V}_{2}^{-}$defect is optically active and an absorption band is found at $0.34 \mathrm{eV}$ due to transitions between internal states ${ }^{8}$. There are no luminescence transitions around $1 \mathrm{eV}$. Further, there has been no luminescence bands to our knowledge correlated with $\mathrm{V}_{3}, \mathrm{~V}_{4}$ and $\mathrm{V}_{5}$. In all these defects it is not possible to pair up all the dangling bonds and they all have deep gap-levels. Such levels can trap carriers and as such the $S=1 / 2$ charged defects can be detected in electron paramagnetic resonance (EPR). These vacancy defects may then be non-radiative centres and remain so when trapped at a dislocation core. Accordingly, they are unlikely to be responsible for the $\mathrm{D}$ optical lines.

Consider now however $\mathrm{V}_{6}$. This then is the first vacancy defect which could luminesce in the $1 \mathrm{eV}$ region. This has six dangling bonds above and six below the hexagon of removed Si atoms (see Figure 1). In this case, all the bonds can be paired up and a defect made whose electronic activity is minimal ${ }^{9-11}$. The reconstruction is symmetric and the resulting symmetry of the centre is $D_{3 d}$. This reconstruction is of course similar to that occurring at a dislocation core. 
Now, the question arises as to the electrical behaviour of the hexavacancy. This has now been answered but in an indirect way ${ }^{11}$. It has been found that neutron irradiated Si containing $\sim 10^{16} \mathrm{~cm}^{-3}$ of hydrogen yields a number of photoluminescent lines linked to hydrogen ${ }^{12}$. Indeed these lines shift in mixtures of hydrogen and deuterium proving that these atoms are part of the optical centre of the defect. Moreover, the shifts of the lines demonstrate that one of these centres, $\mathrm{B}_{41}$, contains two equivalent $\mathrm{H}$ atoms. What is the origin of this line?

Careful annealing studies demonstrate that in neutron irradiated material a luminescence band at $1.107 \mathrm{eV}$ called the J-line occurs in a temperature range up to about $450{ }^{\circ} \mathrm{C}$ when multivacancy defects, including for example $\mathrm{V}_{5}$, are known to occur. This centre is believed to be intrinsic as its production increases with irradiation without saturation. The defect can be aligned under stress in a similar manner to the divacancy suggesting it is a multivacancy centre. Moreover, the J-centre is not formed when hydrogen is present, but the other H-related PL centres are then detected. It seems then that hydrogen is reacting with the J-centre leading to the hydrogen related defects.

A study of the effect of a magnetic field on the J-line has shown that the defect is trigonal ${ }^{13}$. Now a pair of equivalent atoms can only be added to a trigonal centre if the defect possesses further symmetries such as inversion. The effective symmetry of the Jcentre is then $D_{3 d}$ and this is the same symmetry as the hexavacancy. No interstitial defect has been constructed which has this symmetry and we conclude, that the J-line is due to the hexavacancy. The calculations ${ }^{11}$ we have carried out support the view that a strong reconstruction occurs between the twelve dangling bonds of $\mathrm{V}_{6}$ and that only optical transitions in the vicinity of the band gap are possible.

In summary, the hexavacancy is the only vacancy aggregate known to be optically active in the $1 \mathrm{eV}$ region but of course there are many interstitial defects known to luminescence in this energy region.

\section{INTERSTITIAL CLUSTERS}

From a theoretical viewpoint, analysing the structure of self-interstitial clusters has been a highly topical area. There are many papers dealing with possible structures but regrettably there has been few serious attempts to try to relate the centres to observed defects. Here we adopt a different strategy.

Most workers would agree that an interstitial defect whose dangling bonds can be reconstructed would be particularly stable. This is the case for the tetra-interstitial $\mathrm{I}_{4}$ defect built out of four [100] split-interstitials ${ }^{10,14,15}$.

The split-interstitial shown in Figure 2 has two orthogonal $p$-orbitals which can be considered as dangling bonds. These readily react with other defects. Indeed in Si we know that the interstitial complexes with hydrogen yielding a passive defect $\mathrm{IH}_{2}{ }^{16}$. Thus, even if the neutral interstitial $\mathrm{I}_{1}$ had a different structure, it is clear that $\mathrm{IH}_{2}$ is a perturbed [100] split-interstitial saturated with $\mathrm{H}$. Now if we bring together two neighbouring splitinterstitial defects as in Figure 3, then one pair of dangling bonds can be reconstructed. The reconstructed bond bridges the two split-interstitials along [011].

We now show the other pair of dangling bonds along [01] can also be eliminated. Suppose we bring up another di-interstitial on a neighbouring (011) plane. This like the first will 
possess two dangling bonds and one reconstructed bond. But placing then next to one another then allows us to pair up the four dangling bonds (aligned along [011] ) forming altogether four reconstructed bonds parallel to $\langle 011\rangle$ as shown in Figure 4. Such a defect has been suggested by Aria et al. ${ }^{14}$ as a low energy interstitial cluster in Si. However, the same defect has been suggested many times and the earliest reference we are aware of is due to Humble ${ }^{17}$ who argued it is a building block for an extended defect in diamond called the platelet. Muto and Takeda ${ }^{18}$ have suggested that an extended (100) interstitial platelets in Ge has the same structure.

It is clear that the stability of the defect arises from the elimination of dangling bonds and this raises a crucial question. If there are no dangling bonds in the $\mathrm{I}_{4}$ defect, then surely, like reconstructed partials, there will be no gap states and the defect will be electrically, magnetically and optically inert. However, our calculations ${ }^{15}$ show that the defect is not inert and has a level deep donor level in the lower half of the band gap. The question now arises is why should this occur.

To answer this, let us consider a tight binding model where an atomic orbital is coupled to neighbouring orbitals in a lattice of coordination $z$. If the Hamiltonian matrix elements between neighbouring coupling atoms is $V$, then it is easy to show there is a band of states stretching from $-z|V|$ to $z|V|$. Now imagine an interstitial defect inserted in the middle of the lattice but having the same coordination as the bulk atoms. These bonds will be compressed and consequently the magnitude of $|V|$ will be locally increased by a factor say of $f$. It can be shown that the range for the energy eigenvalues is now increased to $-z f|V|$ to $z f|V|$. Thus we expect states to move out of the valence band into the gap arising from these strengthened bonds. The same conclusion is reached if the effect of the interstitial is to locally increase the coordination $z$. In this way we can persuade ourselves that interstitial defects can give rise to states in the lower half of the gap close to $E_{v}$. Of course, there are many terms not considered in this simple tight binding Hamiltonian. There will be changes to bond angles and to the diagonal matrix elements and these will also cause a shift of the defect levels. Nevertheless the best calculations we can do is to use an ab initio density functional method to deduce the structure of the defect and then to evaluate its ionisation energy. This is the energy necessary to remove a electron from the defective crystal. Our calculations showed that this is almost the same as the ionisation energy of the interstitial carbon defect $\mathrm{C}_{i}$. Thus we conclude ${ }^{19}$ that the defect must have a similar donor level as $\mathrm{C}_{i}$ which is known from deep level transient spectroscopy (DLTS) studies to lie at $E_{v}+0.28 \mathrm{eV}$. The implication is that $\mathrm{I}_{4}$ in $\mathrm{p}$-Si should be present as as an ionised species $\mathrm{I}_{4}^{+}$and thus EPR active. We now ask whether an EPR signal has been found with properties expected of $\mathrm{I}_{4}$.

The B3 EPR centre $(S=1 / 2)$ has been investigated by Brower ${ }^{20}$. This centre is detected in neutron irradiated p-type FZ-Si annealed between 170 to $500{ }^{\circ} \mathrm{C}$. It possesses $D_{2 d}$ symmetry - the same as $\mathrm{I}_{4}$. The defect is clearly very stable and not related to oxygen or carbon as $10^{16} \mathrm{~cm}^{-3} \mathrm{~B} 3$ centres can be formed - well above the concentrations of these elements. Brower makes the point that the unusual symmetry is not the result of motional averaging as in a Jahn-Teller distortion of a vacancy centre, but rather the symmetry is intrinsic. Hyperfine interactions on two atoms along the principal [100] axis have been resolved. This implies that the core of the defect is empty for otherwise interaction with a unique atom would have been detected. Of course, all these properties are expected of $\mathrm{I}_{4}$, although in fact Brower suggests that the defect is a di-interstitial. The donor level has been placed at 
$E_{v}+0.29 \mathrm{eV}$ by Mukashev et al. ${ }^{21}$ who noted a correlation between B3 and the $E_{v}+0.29 \mathrm{eV}$ level detected in DLTS. This agrees remarkable well with our estimate of the donor level.

Thus the properties of B3 are entirely in agreement with the calculations. But is the defect optically active? We believe there is a link with a prominent optical centre called I3 or $\mathrm{X}(1.0398 \mathrm{eV})$. The notation I3 is unfortunate and may be confusing with the tri-interstitial $\mathrm{I}_{3}$ and so we shall refer to the optical centre as X.

The effect of annealing irradiated or implanted Si and following the evolution of photoluminescent $(\mathrm{PL})$ centres is instructive. Terashima et al. ${ }^{22}$ implanted $\mathrm{Cz}-\mathrm{Si}$ with $\mathrm{P}$ and $\mathrm{B}$ ions and subsequently annealed the samples between 200 and $700^{\circ} \mathrm{C}$. At $200^{\circ} \mathrm{C}$, a strong PL line called $\mathrm{I} 1$ or $\mathrm{W}$ at $1.018 \mathrm{eV}$ was detected. Its intensity, relative to B-related bound exciton, first increased until about $200{ }^{\circ} \mathrm{C}$ and then decreased and the line disappeared by $500{ }^{\circ} \mathrm{C}$. As the $\mathrm{W}$ signal diminished, the intensity of the $\mathrm{X}$-line increased. This was not present at $200^{\circ} \mathrm{C}$ but became apparent at $300^{\circ} \mathrm{C}$, and its intensity increased until about around $450{ }^{\circ} \mathrm{C}$. It subsequently decreased and vanished by $600^{\circ} \mathrm{C}$. These two lines dominated the spectrum but there were less intense bands due to $\mathrm{I} 2$ at $1.080 \mathrm{eV}$ - a trigonal defect containing two $\mathrm{B}$ atoms $^{13}$ - and a line at $1.097 \mathrm{eV}$. Interestingly, annealing in an atmosphere containing $\mathrm{Cu}$, led a similar spectrum but an additional band at $0.992 \mathrm{eV}$ was detected which disappeared around $550^{\circ} \mathrm{C}$.

Similar results were reported by Awadelkelkarim et al. ${ }^{23}$. Here, both $\mathrm{Cz}-\mathrm{Si}$ and FZ-Si were implanted with $\mathrm{C}$ and $\mathrm{O}$ and annealed to $500{ }^{\circ} \mathrm{C}$. Besides the well known carbon and oxygen related lines: $\mathrm{P}(0.7671 \mathrm{eV}), \mathrm{H}(0.9254 \mathrm{eV}), \mathrm{L}(1.0038 \mathrm{eV}), \mathrm{G}(0.9694 \mathrm{eV})$, and $\mathrm{C}$ $(0.7890 \mathrm{eV})$, the $\mathrm{X}$ and $\mathrm{W}$ lines were also detected. Moreover, in FZ-Si these, together with the Y-line at $1.08 \mathrm{eV}$, were the principal emissions. Again the $\mathrm{W}$-line although detected up to $350{ }^{\circ} \mathrm{C}$, vanished at $400{ }^{\circ} \mathrm{C}$ while the $\mathrm{X}$-line was observed from about 250 to $500{ }^{\circ} \mathrm{C}$.

In summary, the annealing data in irradiated FZ-Si indicate that the $\mathrm{W}$-centre is formed at an earlier stage than $\mathrm{X}$ and is stable until about $350^{\circ} \mathrm{C}$ but as this disappears, the $\mathrm{X}$ centre evolves and is stable until about $500{ }^{\circ} \mathrm{C}$. Thus it is inferred that the $\mathrm{W}$ and $\mathrm{X}$ lines are related to intrinsic defects and the $\mathrm{X}$ centre is a larger aggregate than $\mathrm{W}$. Both optical signals are seen in absorption as well as luminescence and represent transitions between gap states. This distinguishes them from bound exciton transitions found, for example, in the J-centre.

Now uniaxial stress measurements have been carried out on both of these centres . For $\mathrm{X}$, the line is split into two by $\langle 100\rangle$ stress with one component being detected with an $E$ field parallel to stress axis and the other perpendicular to $\mathrm{it}^{24}$. Similarly, the X-line is split under $\langle 110\rangle$ but not by $\langle 111\rangle$ stress. It is clear that this behaviour is consistent with $D_{2 d}$ symmetry if the optical transition occurs between either $a_{1}$ and $b_{2}$ or $a_{2}$ and $b_{1}$ states. Stressing along any $\langle 111\rangle$ direction in Figure 4 would produce equivalent defects. However, the same stress pattern is found if the centre has $T_{d}$ symmetry, but in this case an electric dipole transition could only occur if one of the states had $t_{2}$ symmetry. The effect of stress is then reflected in the splitting of the $t_{2}$ manifold and one expects the vibronic structure to be associated with a $t_{2}$ or $e$ vibrational mode. However, there is no evidence that these modes are involved and thus the symmetry of the defect is is believed to be $D_{2 d}$.

Given the thermal stability of the X-defect and its symmetry it is tempting to identify it with $\mathrm{I}_{4}$. However, one possible problem is that the stress sensitivity of the defect is anomalously large. Under [100] stress, the line splits by $117 \mathrm{meV} / \mathrm{GPa}$ which is greater 
than the splitting of the conduction band $\left(92.5 \mathrm{meV} / \mathrm{GPa}^{25}\right)$. Of course this may reflect the softness of the reconstructed bonds but is contrary to the 'rule' that interstitial defects are elastically hard. The transition energy is $0.1 \mathrm{eV}$ greater than the depth of the donor level below the conduction band edge and as it is an internal transition, we suppose that lower lying states are involved.

Further work is necessary to confirm that the defects are the same. We know from the EPR investigations that the reorientation energy of B3 is $2.3 \mathrm{eV}$ and if this energy could be measured for the $\mathrm{X}$ centre, then it would provide irrefutable evidence for the identity of the defects.

Assuming that $\mathrm{X}$ is then $\mathrm{I}_{4}$ it is clear that the $\mathrm{W}$-line possess fewer interstitials, and one possibility suggested previously ${ }^{26}$ is that it is a trigonal form of $\mathrm{I}_{3}$ shown in Figure 5 . The symmetry of the defect is known to be trigonal ${ }^{27}$. The main evidence for the assignment comes from the calculated local vibrational modes which are close to a phonon replica found at $564 \mathrm{~cm}^{-1}$. This undergoes large isotopic shifts with ${ }^{29} \mathrm{Si}$ and ${ }^{30} \mathrm{Si}$. These imply that the vibration is due to a compressed Si-Si bond lying on the trigonal axis so that the vibrational mode lies above the Raman and is localised on one or two Si atoms.

\section{THE D LUMINESCENCE BANDS}

These bands are believed to be related to dislocations as they increase in intensity with dislocation density. They are seen in both $\mathrm{Cz}$ and FZ material irrespective of type, and are stable to $1000^{\circ} \mathrm{C}$. They exhibit strong polarisation along the dislocation line direction ${ }^{28}$. D1 and D2 are initially enhanced by oxygen ${ }^{29,30}$ and low concentration of transition metal impurities such as $\mathrm{Cu}^{31,32}$. However, larger concentrations of $\mathrm{Cu}$ suppress the bands. The effect of hydrogen has been controversial. Initial reports suggested a suppression of the bands but later work indicated that the intensity of some bands could be increased ${ }^{33}$. Similar lines are seen in SiGe epilayers, up to at least $800^{\circ} \mathrm{C}$ although their energies are red-shifted by about $0.1 \mathrm{eV}^{34}$.

It is clear that impurities have an effect on the bands and we suppose that the impurities, or the $\mathrm{SiGe-Si} \mathrm{interface} \mathrm{generate} \mathrm{interstitials,} \mathrm{or} \mathrm{less} \mathrm{likely} \mathrm{vacancies,} \mathrm{which} \mathrm{are} \mathrm{subsequently}$ trapped by the dislocation. Small clusters of these defects are not thermally stable and break up or move rapidly along the core until more stable larger clusters are made. Clearly, if $\mathrm{I}_{4}$ and $\mathrm{V}_{6}$ are stable at $500^{\circ} \mathrm{C}$ as point defects, then their stability is likely to be enhanced at a dislocation core. This follows as the strain surrounding the defect can be relieved at the dislocation. However, their structure is likely to be perturbed with consequent shifts of their electronic levels. If the levels move towards mid-gap, then we anticipate a downward shift in the emission energy, and an increase in the thermal stability of the luminescence. We thus identify the D-band luminescence with these, or even larger, clusters.

There are many questions with the hypothesis advanced here: the binding energies of the clusters with the core need to be evaluated; the perturbation to their energy levels caused by the core; the relationship with the DLTS levels at $E_{c}-0.2,0.3,0.5$ and $0.54 \mathrm{eV}$ observed in $\mathrm{n}_{-} \mathrm{Si}^{35}$, and the $E_{v}+0.47 \mathrm{eV}$ level detected in $\mathrm{p}-\mathrm{Si}$, as well as the dislocation related EPR centres, $\mathrm{K} 1$ to $\mathrm{K} 5^{36}$. In addition the roles of oxygen, hydrogen and $\mathrm{Cu}$ need to be investigated. We hope to discuss these aspects in a future publication. 


\section{ACKNOWLEDGEMENT}

SÖ thanks NFR and TFR, and RJ thanks ENDEASD for financial support. We also thank Gordon Davies for useful discussions. 


\section{REFERENCES}

${ }^{1}$ N. A. Drozdov, A. A. Patrin, V. D. Tkachev, Sov. Phys., JETP Lett. 23, 597 (1996).

${ }^{2}$ E. O. Sveinbjornsson, J. Weber, Appl. Phys. Lett. 69, 2686 (1996).

${ }^{3}$ R. Jones, Mat. Sci. Eng. B 71, 24-9, (2000).

${ }^{4}$ V. Higgs, E. C. Lightowlers, E. A. Fitzgerald, Y.-H. Xie, J. Silverman, J. Appl. Phys. 73, 1952 (1993).

${ }^{5}$ E. A Steinman, and H. G. Grimmeiss, Semicon. Sci. Technol. 13, 124 (1998).

${ }^{6}$ G. P. Watson, J. L. Benton, and Y. H. Xie, E. A. Fitzgerald, J. Appl. Phys. 83, 3773 (1998).

${ }^{7}$ G. D. Watkins, and J. W. Corbett, Phys. Rev. B 9, 4351 (1974).

${ }^{8}$ J. H. Svensson,B. G. Svensson, and B. Monemar, Phys. Rev. B 38, 4192 (1988).

${ }^{9}$ Chadi D. J. and Chang, K. J, Phys. Rev. B 38, 1523 (1988).

${ }^{10}$ S. K. Estreicher, J. L. Hastings, and P. A. Fedders, Appl. Phys. Lett. 70, 432 (1997).

${ }^{11}$ B. Hourahine, R. Jones, A. N. Safonov, S. Öberg, and P. R. Briddon, and S. K. Estreicher, Phys. Rev. B 61, May 2000

${ }^{12}$ A. N. Safonov, E. C. Lightowlers, and G. Davies, Phys. Rev. B 56, 15517 (1997).

13 J. Weber, Physica B 116, 195 (1983).

${ }^{14}$ N. Aria, S. Takeda, and M. Kohyama, Phys. Rev. Lett. 78, 4265 (1997).

${ }^{15}$ B. J. Coomer, J. P. Goss, R. Jones, S. Öberg, and P. R. Briddon, submitted.

${ }^{16}$ M. Budde, B Bech Nielsen, P. Leary, J. Goss, R. Jones, P. R. Briddon, and S. Öberg, Phys. Rev. B 574397 (1998).

${ }^{17}$ P. Humble, Proc. Roy. Soc. Lond. A 381, 65 (1982).

18 S. Muto, and S. Takeda, Phil. Mag. Lett. 72, 99 (1995).

${ }^{19}$ A. Resende, R. Jones, S. Öberg, and P. R. Briddon, Phys. Rev. Lett. 82, 2111 (1999).

${ }^{20}$ K. L. Brower, Phys. Rev. B 14, 872 (1975).

${ }^{21}$ B. N. Mukashev, A. V. Spitsyn, N. Kukuoka, and H. Saito, Jpn. J. Appl. Phys. 21, 399 (1982).

${ }^{22}$ K. Terashima, T. Ikarashi, M. Watanabe, and T. Kitano, Mater. Sci. Forum 258-263, 587 (1997).

${ }^{23}$ O. O. Awadelkelkarim, A. Henry, B. Monemar, J. L. Lindström, Y. Zhang, and J. W. Corbett, Phys. Rev. B 42, 5635 (1990).

${ }^{24}$ Z. Cienchanowska, G. Davies, and E. C. Lightowlers, Solid State Comm. 49, 427 (1984).

${ }^{25}$ L. D. Laude, F. H. Pollack, and M. Cardona, Phys. Rev. B 3, 2623 (1971).

${ }^{26}$ B. J. Coomer, J. P. Goss, R. Jones, S. Öberg, and P. R. Briddon, Physica B 273-4, 505 (1999).

${ }^{27}$ V. D. Tkachev, and A. V. Mudryi, J. Appl. Spectrosc. 29, 1485 (1979).

${ }^{28}$ M. Suezawa, and K. Sumino, J. de Physique, C4 44, 133 (1983).

${ }^{29}$ O. V. Feklisova, G. Mariani-Regula, B. Pichaud, E. B. Yakimov, Physica Status Solidi A-Applied Research 171, 341 (1999).

${ }^{30} \mathrm{~V}$. Higgs, in Early stages of oxygen precipitation in silicon, Vol. 17 of NATO ASI - 3. High Technology, edited by R. Jones (Kluwer Accademic Publishers, Dordrecht, 1996), p. 469.

${ }^{31}$ V. Higgs, M. Golding, A. Brinklow and P. Knightley, Appl. Phys. Lett. 60, 1369 (1992).

32 T. Okuyama, M. Suezawa, I. Yonenaga, and K. Sumino, Mat. Sci. Forum 196-201, 1213 (1995).

${ }^{33}$ T. Sekiguchi, V. V. Kveder, K. Sumino, J. Appl. Phys. 76, 7882 (1994). 
${ }^{34}$ P. M. Mooney, and K. Shum, Mat. Sci. Forum 258-73, 151 (1997).

${ }^{35}$ P. Omling, E. R. Weber, L. Montelius, H. Alexander and J. Michel, Phys. Rev. B 32, $6571(185)$.

${ }^{36}$ E. R. Weber, and H. Alexander, J. Phys. (Paris) Colloq. 44, C4-319 (1983). 


\section{FIGURES}
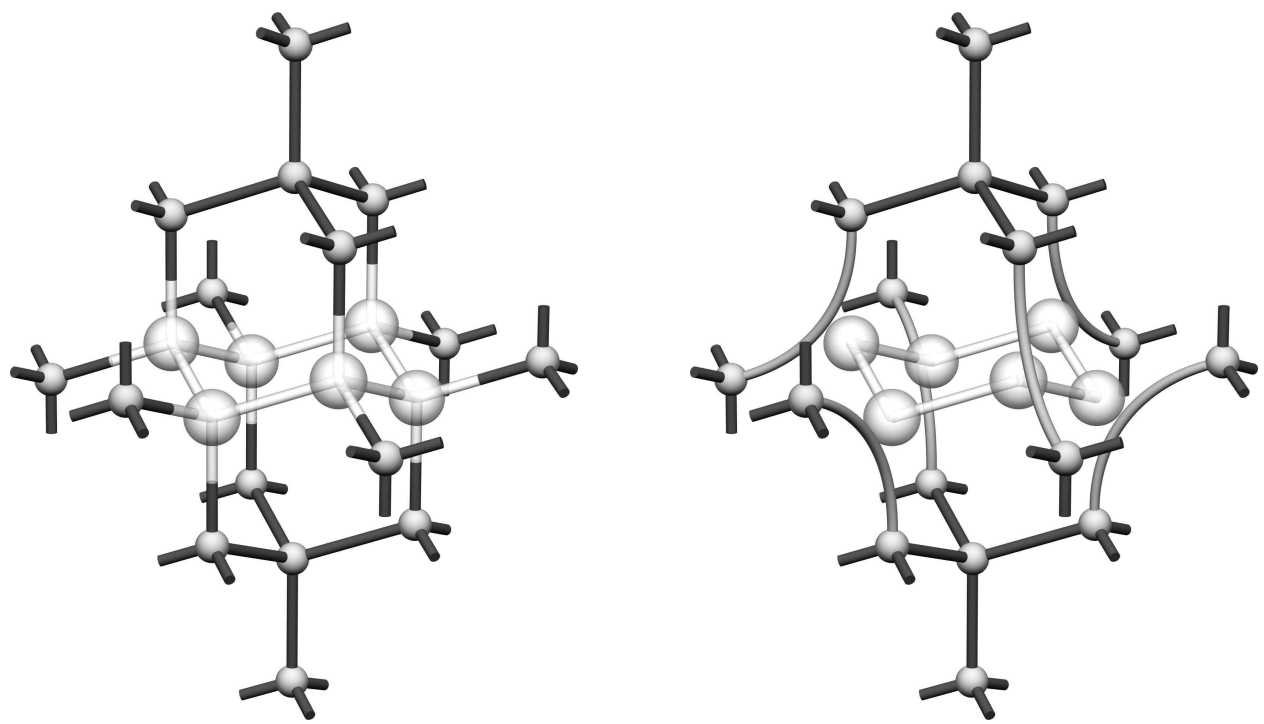

FIG. 1. Ideal and reconstructed hexavacancy defects. The transparent spheres represent the vacancy sites.

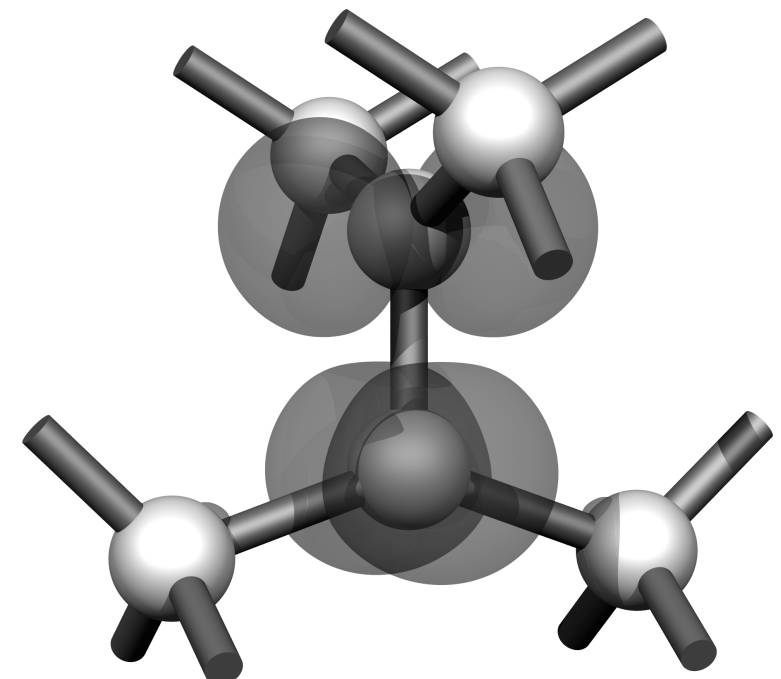

FIG. 2. Schematic of the [100] split-interstitial. Also indicated are the two $p$-orbitals associated with the three-fold-coordinated atoms. 


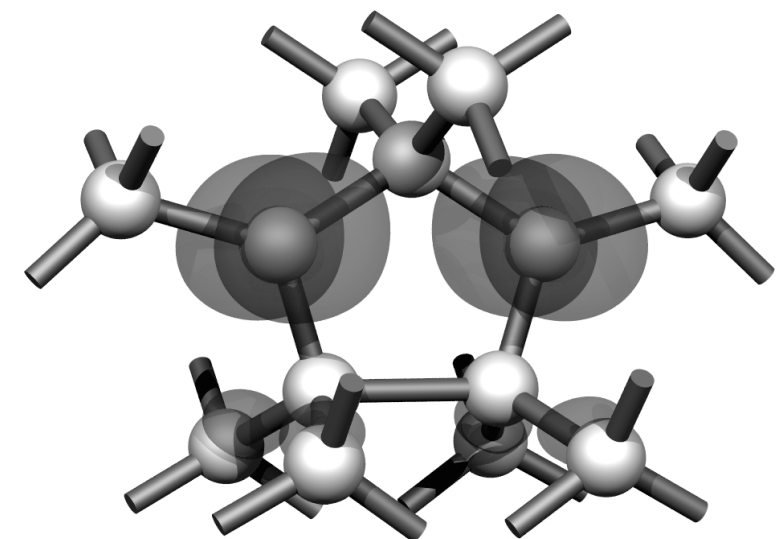

FIG. 3. The reconstructed self-interstitial pair. This complex also possesses two three-fold coordinated atoms with $p$-orbitals.

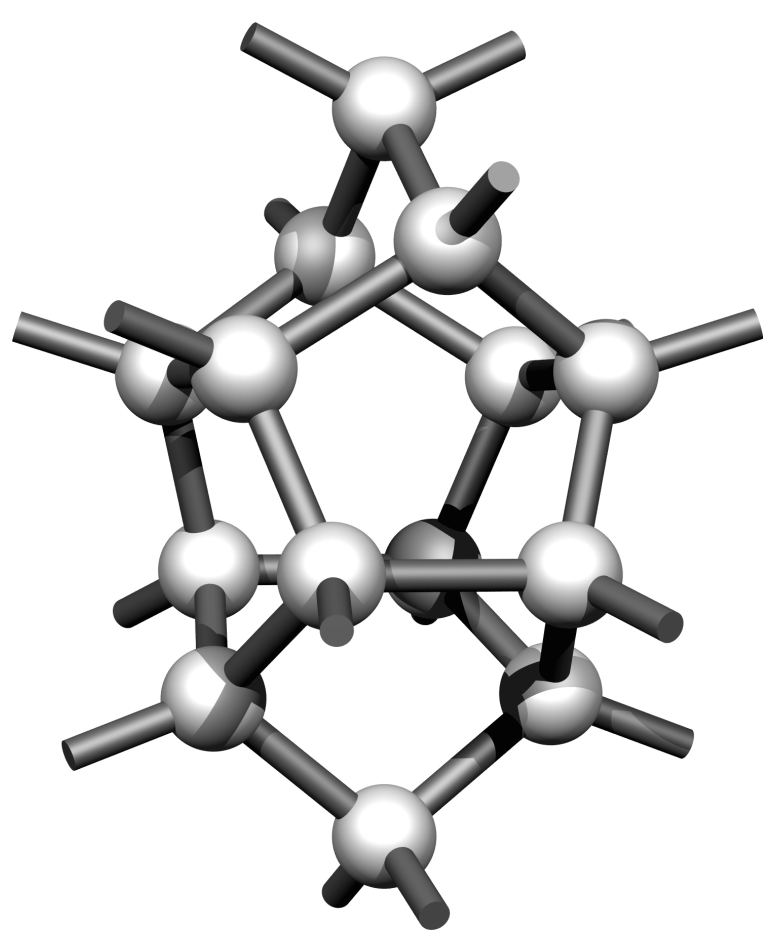

FIG. 4. Schematic of the fully reconstructed $\mathrm{I}_{4}$ defect. 


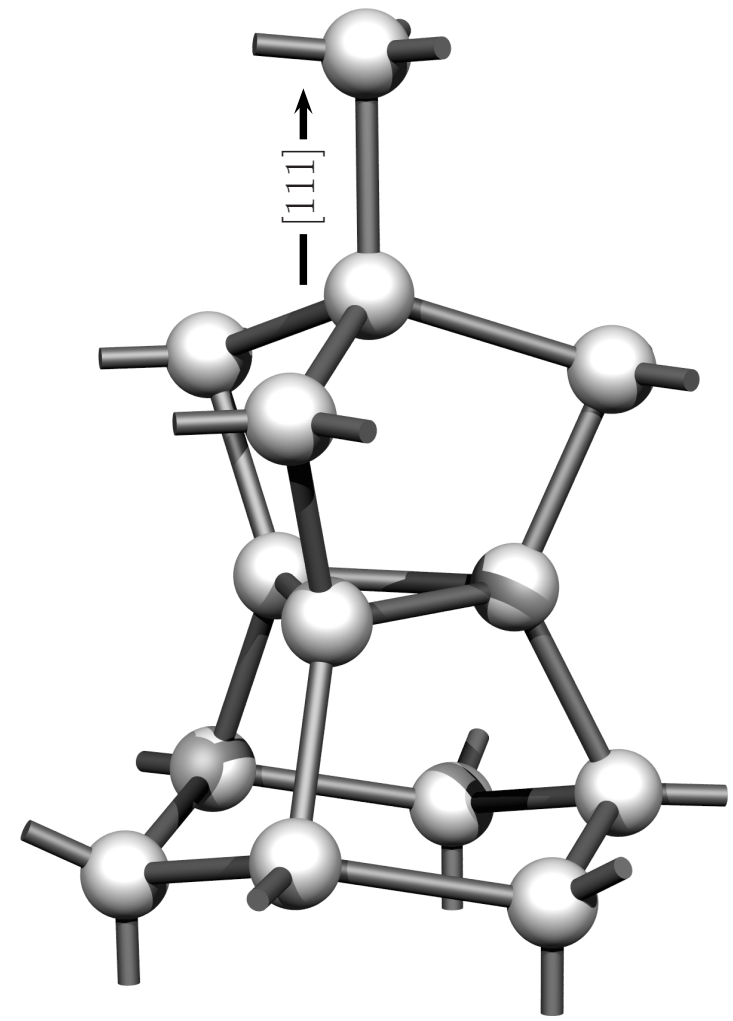

FIG. 5. Trigonal tri-interstitial defect. 